\title{
Dupla jornada de trabalho na enfermagem: dificuldades enfrentadas no mercado de trabalho e cotidiano laboral ${ }^{a}$
}

\author{
Double working hours in nursing: difficulties faced in the labor market and daily work \\ Doble jornada en enfermería: dificultades en el mercado laboral y el trabajo diario
}

\begin{abstract}
Samira Silva Santos Soares ${ }^{1}$ (1)
Marcia Tereza Luz Lisboa ${ }^{1}$ (D) Ana Beatriz Azevedo Queiroz ${ }^{1}$ (C)

Karla Gualberto Silva ${ }^{1}$ (D)

Jandra Cibele Rodrigues de Abrantes

Pereira Leite ${ }^{1}$ (1)

Norma Valéria Dantas de Oliveira Souza ${ }^{2}$ (1)

1. Universidade Federal do Rio de Janeiro, Escola de Enfermagem Anna Nery. Rio de Janeiro, RJ, Brasil.

2. Universidade do Estado do Rio de Janeiro, Faculdade de Enfermagem. Rio de Janeiro, RJ, Brasil.
\end{abstract}

Autor correspondente

Samira Silva Santos Soares

E-mail: samira_opg@hotmail.com

Recebido em 10/09/2020.

Aprovado em 23/12/2020.

DOl:https://doi.org/10.1590/2177-9465-EAN-2020-0380

\begin{abstract}
Resumo
Objetivo: compreender por meio da lexicografia básica os vocabulários mais frequentes expressos por trabalhadores de enfermagem sobre a experiência de ter uma dupla jornada de trabalho. Método: pesquisa qualitativa, realizada com 30 trabalhadores de enfermagem com dupla jornada de trabalho captados pela técnica snowball, entre janeiro e março de 2019 na cidade de Eunápolis, Bahia, Brasil. Os dados foram obtidos por meio de uma entrevista semiestruturada, processados com o auxílio do software Interface de $\mathrm{R}$ pour les Analyses Multidimensionnelles de Textes et de Questionnaires (IRAMUTEQ ${ }^{\circledR}$ ). A partir da nuvem de palavras deu-se a análise lexical. Resultado: os léxicos mais frequentes identificados no corpus foram "não" e "gente". Conclusão e implicações para a prática: os vários "nãos" evocados pelos participantes apontam que a experiência de trabalho é marcada por negatividade, restrições e sofrimento, fato que coloca em risco a saúde dessa "gente", ou seja, desse coletivo profissional, suas relações sociais e até mesmo a qualidade da assistência prestada ao cliente. A compreensão desse contexto é fundamental para produzir reflexões que contribuam para a construção de políticas públicas voltadas à valorização e proteção dos trabalhadores de enfermagem bem como para reforçar a indispensável necessidade da enfermagem se fortalecer como classe trabalhadora.
\end{abstract}

Palavras-chave: Enfermagem; Jornada de Trabalho; Mercado de Trabalho; Saúde do Trabalhador; Pesquisa qualitativa.

\begin{abstract}
Objective: Understand, through basic lexicography, the most frequent vocabularies expressed by nursing workers on the experience of having a double workday. Method: Qualitative research, carried out with 30 nursing workers with double working hours captured by the snowball technique, between January and March 2019, in the city of Eunápolis, Bahia, Brazil. The data were obtained through a semi-structured interview, processed with the aid of the software Interface de pour pour Analyzes Multidimensionnelles de Textes et de Questionnaires (IRAMUTEQ ${ }^{\circledR}$ ). From the word cloud, lexical analysis took place. Result: The most frequent lexicons identified in the corpus were "no" and "people". Conclusion and implications for the practice: The various "nos" evoked by the participants point out that the experience is marked by negativity, restrictions and suffering, a fact that puts the health of the "people" at risk, that is, of this professional group, their social relations and even the quality of the assistance provided to the customer. Understanding this context is essential to produce reflections that contribute to the construction of public policies aimed at valuing and protecting nursing workers, as well as to reinforce the indispensable need for nursing to strengthen as a working class.
\end{abstract}

Keywords: Nursing; Work Hours; Job Market; Occupational Health; Qualitative research; Software.

\section{REsumen}

Objetivo: Comprender, a través de la lexicografía básica, los vocabularios más frecuentes expresados por los trabajadores de enfermería sobre la experiencia de tener una doble jornada laboral. Método: Investigación cualitativa, realizada con 30 trabajadores de enfermería con doble jornada capturada por la técnica de bola de nieve, entre enero y marzo de 2019, en la ciudad de Eunápolis, Bahía, Brasil. Los datos se obtuvieron a través de una entrevista semiestructurada, procesada con la ayuda del software Interface de pour pour Analyzes Multidimensionnelles de Textes et de Questionnaires (IRAMUTEQ ${ }^{\circledR}$ ). A partir de la nube de palabras, se realizó el análisis léxico. Resultado: Los léxicos más frecuentes identificados en el corpus fueron "no" y "personas". Conclusión e implicaciones para la práctica: los distintos "no" evocados por los participantes señalan que la experiencia laboral está marcada por la negatividad, las restricciones y el sufrimiento, hecho que pone en riesgo la salud de estas "personas", es decir, de este grupo professional, su relaciones sociales e incluso la calidad de la asistencia prestada al cliente. Comprender este contexto es fundamental para producir reflexiones que contribuyan a la construcción de políticas públicas orientadas a la valoración y protección de los trabajadores de enfermeira, así como a reforzar la indispensable necesidad de que la enfermería se fortalezca como clase trabajadora.

Palabras-clave: Enfermería; Horas de Trabajo; Mercado de Trabajo; Salud laboral; investigación cualitativa. 


\section{INTRODUÇÃO}

A jornada de trabalho é definida como o lapso temporal diário em que o trabalhador se coloca à disposição do empregador e vende sua força de trabalho em virtude de um determinado contrato de trabalho. ${ }^{1,2}$ No âmbito da enfermagem, em função dos baixos salários que historicamente são recebidos pela categoria, é comum que seus trabalhadores realizem uma dupla jornada de trabalho, ${ }^{3}$ condição vivenciada por aqueles que assumem mais de um vínculo laboral, geralmente, na expectativa de um melhor retorno financeiro pelo trabalho desempenhado.

Tal fato, no entanto configura-se em um processo de intensificação do trabalho e que precisa ser repensando, tendo em vista que a realização de longas jornadas de trabalho está associada ao aumento de ocorrências adversas na saúde e que pode culminar no adoecimento físico, mental e social dos trabalhadores e ainda afetar a segurança dos pacientes sob seus cuidados. ${ }^{4}$

Christophe Dejours, médico do trabalho, psiquiatra e psicanalista e maior representante do campo da Psicodinâmica do Trabalho assevera que o trabalho nunca é neutro em relação à saúde,${ }^{5}$ podendo promovê-la ou conduzir ao adoecimento, daí a importância de compreender como o trabalhador lida com o trabalho e como este se torna fator determinante e condicionante no processo saúde/doença.

Desse modo, cientes de que não há até o momento, um dispositivo legal que regule a nível nacional sobre a jornada de trabalho no contexto da enfermagem, ${ }^{6}$ e considerando que esses profissionais estão inseridos em um mercado de economia capitalista, que pautado em doutrinas políticas e econômicas as quais têm por base o neoliberalismo e os torna ainda mais vulneráveis aos processos de desgaste e adoecimento, ${ }^{7-9}$ tornase fundamental investigar sobre essa temática.

Ademais, estudos acerca da percepção dos trabalhadores de enfermagem com dupla jornada de trabalho (profissional) sobre o seu cotidiano de trabalho e o mercado de trabalho são escassos, necessitando, portanto, de mais investigações para elucidação desse fenômeno. Desse modo, esta pesquisa trará como contribuição uma breve reflexão acerca de tal temática e sobre o mercado de trabalho onde os trabalhadores de enfermagem se encontrem inseridos. $E$, ao utilizar o software Iramuteq ${ }^{\circledR}$ o estudo contribui para divulgar essa possibilidade de aplicação no contexto da pesquisa qualitativa.

Diante do exposto, este estudo teve como objetivo compreender por meio da lexicografia básica os vocabulários mais frequentes expressos por trabalhadores de enfermagem sobre a experiência de ter uma dupla jornada de trabalho.

\section{MÉTODO}

Estudo exploratório-descritivo, estruturado em uma abordagem qualitativa de pesquisa. Realizado com 30 trabalhadores de enfermagem com dupla jornada de trabalho, que foram captados pela técnica snowball (bola de neve), na cidade de Eunápolis, Bahia, Brasil, durante os meses de janeiro a março de 2019.
A aplicação dessa técnica teve como start duas participantes enfermeiras, que atuam no Município do Eunápolis/BA. Na sequência, essas pessoas foram indicando novos participantes para a pesquisa, que recomendavam outras e, assim, criou-se uma "bola de neve" até que a investigadora obteve o número de pessoas suficiente para a constituição da amostra.

Estabeleceu-se como critérios de inclusão para participar do estudo: trabalhadores de enfermagem (enfermeiros, técnicos de enfermagem) com dupla jornada de trabalho em decorrência de ter dois vínculos trabalhistas na área da enfermagem, sendo praticados em instituições do setor público e/ou privado em atividades assistenciais e/ou gerenciais de enfermagem; além de residir no Município de Eunápolis/Ba ou em municípios que integram a mesma região de saúde.

$E$, como critérios de exclusão, elencou-se que fossem trabalhadores de enfermagem (Enfermeiros, técnicos de enfermagem) com dupla jornada de trabalho há menos de um ano consecutivo. Esse critério fundamentou-se no fato de acreditar que o período de um ano é minimamente necessário para que os trabalhadores de enfermagem com dupla jornada de trabalho familiarizem-se com as rotinas necessárias ao cumprimento dessas duas jornadas em instituições diferentes, considerassem as vantagens e desvantagens dessa prática, avaliassem o retorno financeiro e os impactos produzidos por ela no trabalho e na sua vida pessoal.

Antes da coleta de dados propriamente dita, foi feito o contato com o possível participante pelo telefone, com uma breve explanação sobre a pesquisa e verificando a disponibilidade para um encontro presencial. Também se faz importante comentar que houve dez recusas à participação na pesquisa e o motivo alegado foi a indisponibilidade de tempo, devido à extensa jornada de trabalho.

A coleta de dados foi realizada por meio de questionário para caracterização dos participantes e entrevista semiestruturada As entrevistas foram realizadas, conforme disponibilidade do trabalhador e fora dos seus locais de trabalho, após a assinatura do Termo de Consentimento Livre e Esclarecido (TCLE). A gravação das entrevistas foi realizada com a concordância dos respondentes e após transcritas, os textos provenientes foram revisados em relação à ortografia, sem que a essência fosse alterada e foram devolvidas aos participantes para leitura e aprovação do conteúdo descrito. Quanto ao tempo de duração das entrevistas, destaca-se que a mais curta ocorreu num período de 25 minutos e a mais longa teve 90 minutos de duração.

Salienta-se que a coleta de dados foi interrompida, a partir do momento que se considerou atingir a saturação teórica e empírica e, portanto, os dados produzidos mostravam-se satisfatórios e já respondiam às questões norteadoras e ao objetivo proposto. Desse modo, a partir da sexta entrevista com enfermeiros e da décima quarta entrevista com técnicos de enfermagem observou-se a repetição dos dados e que nenhuma nova informação estava surgindo, e que, portanto, a coleta de novos dados acrescentaria poucas informações à discussão. 
A análise lexical dos dados, ocorreu através do software Interface de R pour les Analyses Multidimensionnelles de Textes et de Questionnaires (IRAMUTEQ ${ }^{\circledR}$ ) versão 0.7 alpha 2. O IRAMUTEQ ${ }^{\circledR}$ possibilita cinco tipos de análises: estatísticas textuais clássicas; pesquisa de especificidades de grupos; classificação hierárquica descendente; análises de similitude e nuvem de palavras. Para fins deste estudo, foi utilizada a análise lexical por meio da nuvem de palavras.

Para que os dados fossem submetidos ao IRAMUTEQ ${ }^{\circledR}$ as seguintes etapas foram seguidas:

1. Organização e preparação dos dados, com a transcrição das entrevistas na íntegra, codificação e a disposição dos dados em formato de corpus textual. Por corpus, entende-se todo o material que se pretende analisar com o auxílio do software; e no estudo em questão, o conjunto de entrevistas. Por sua vez, cada entrevista, passa a ser denominada texto. $\mathrm{E}$, os segmentos de texto são os ambientes das palavras, as principais unidades de análise textual desse tipo de software..$^{10}$

2. Leitura de todos os dados para obter a percepção geral das ideias expressas pelos entrevistados;

3. Processamento dos dados através do software IRAMUTEQ ${ }^{\circledR}$, utilizando-se a opção: nuvem de palavras.

A nuvem de palavras é uma análise lexical simples, porém graficamente interessante, pois, a partir dela, as palavras são agrupadas e organizadas em função da sua frequência no corpus textual. Assim, após a etapa de processamento feita pelo software, recorreu-se a análise e interpretação de sentido das palavras nos discursos dos participantes; e ao recuperar os segmentos de texto, onde tais palavras apareceram, o léxico mais frequente passou a fazer sentido em relação ao contexto da discussão e, desse modo, foi possível que a pesquisadora estabelecesse a interpretação dos dados, comparando os achados com a literatura científica que trata sobre o mercado de trabalho da enfermagem e o processo de precarização do trabalho de enfermagem.

$\mathrm{Na}$ apresentação dos resultados, objetivando manter o anonimato dos participantes, no final dos segmentos de texto (ST) foi utilizada a codificação "T" (técnicos de enfermagem) ou "E" (enfermeiros) para representá-los, seguida de um número arábico que indica a sequência cronológica da interlocução realizada.

O estudo foi submetido à Plataforma Brasil e obteve parecer consubstanciado de aprovação pelo Comitê de Ética em Pesquisa da Escola de Enfermagem Anna Nery sob a CAAE 03857318.9.0000.5238 e parecer № 3.085 .492 em 14 de dezembro de 2018. O desenvolvimento do estudo respeitou os preceitos éticos de participação voluntária, esclarecida e consentida de modo que os participantes recrutados foram resguardados mediante a assinatura do TCLE, respeitando-se sua autonomia, dignidade e anonimato, conforme determina a Resolução no 466/2012 da Comissão Nacional de Ética em Pesquisa do Conselho Nacional de Saúde.

\section{RESULTADOS}

Os resultados serão apresentados em duas seções: caracterização dos trabalhadores participantes e análise lexical - por meio da nuvem de palavras.

Quanto a caracterização dos participantes, dos 30 trabalhadores entrevistados, dez eram enfermeiros e 20 técnicos de enfermagem. A maioria dos participantes era do sexo feminino -23 indivíduos $(76,7 \%)$. A idade dos participantes variou entre 26 a 67 anos, com média de 38 anos. Quanto à autodeclaração de cor/raça, segundo nomenclatura do Instituto Brasileiro de Geografia e Estatística (IBGE) a maioria (22 entrevistados $73,3 \%$ ) considerava-se preto ou pardo.

Todos os participantes tinham dois vínculos de trabalho na enfermagem (17 eram concursados pelo município em, pelo menos, um desses vínculos, 11 tinham os dois vínculos por meio de contratos temporários, e dois possuíam contrato por tempo indeterminado). Ao considerar o tempo de atuação na área de enfermagem, 23 participantes (76,7\%) trabalhavam na área por período superior a 5 anos e 20 (66,7\%) também vivenciavam uma dupla jornada de trabalho por período equivalente. A renda líquida declarada pelos participantes da pesquisa, ao considerarem o somatório dos salários recebidos em decorrência dos seus dois vínculos de trabalho, aponta que 17 técnicos de enfermagem com dupla jornada de trabalho, ou seja, $85 \%$ dos participantes dessa categoria, recebem entre dois e quatro salários mínimos e oito enfermeiros ( $80 \%$ dos entrevistados), recebem entre quatro e dez salários mínimos.

Pelo método de nuvem de palavras, foi possível identificar que o léxico "não" foi o que obteve a maior frequência no corpus, sendo evocado 1.610 vezes. Em seguida, identificou-se a palavra "gente" com 743 evocações. A figura 1, que apresenta a nuvem de palavras exibida pelo IRAMUTEQ ${ }^{\circledR}$ posiciona as palavras aleatoriamente, de forma que as palavras mais frequentes aparecem maiores que as demais, de modo a deixar em evidência o seu destaque no corpus. Tal situação é o caso das palavras "não" e "gente" que aparecem em realce no centro da imagem.

Ao recuperar os segmentos de texto onde a palavra "não" aparece, fica evidente que as discussões sobre a dupla jornada de trabalho se referem a duas dimensões as quais estão explicitadas a seguir.

A primeira dimensão aponta os motivos relatados por esses trabalhadores que os faz adotar uma dupla jornada de trabalho. Esses motivos estabelecem relação com mercado de trabalho da enfermagem. Há relatos sobre a desvalorização da categoria, os baixos salários, a falta de um piso salarial digno, e a fragilidade dos vínculos de trabalho como fatores que contribuem para a perpetuação das duplas jornadas de trabalho entre os profissionais da categoria, que: "não estariam em dois empregos, se tivessem um salário digno", "não saem de um emprego com medo de perder o outro", "não se sentem reconhecidos e valorizados na profissão", "não querem manter dois empregos por muito tempo", "não podem parar por enquanto", "não querem seguir na carreira da enfermagem". 


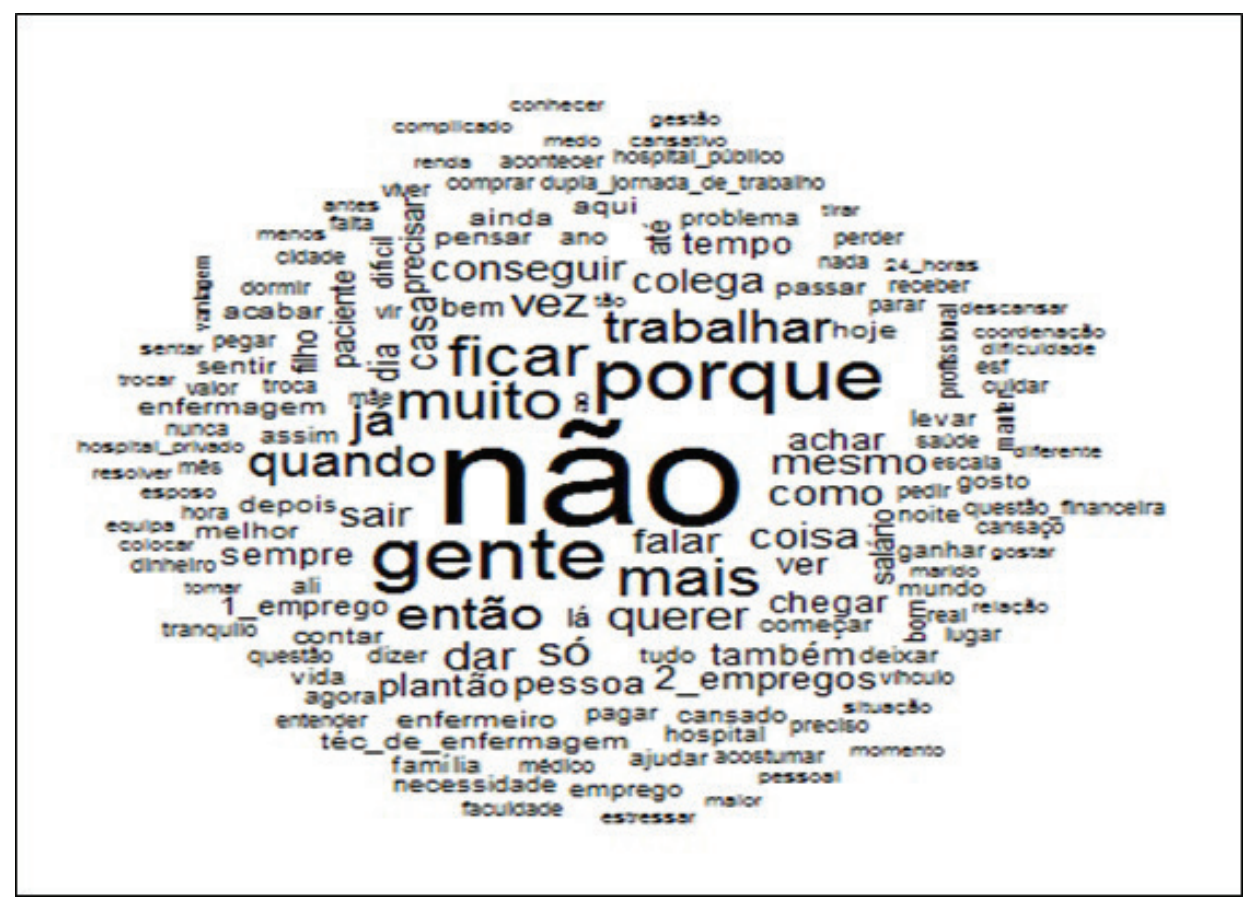

Figura 1. Nuvem de Palavra

Fonte: As autoras (2019), organizado com base no software IRAMUTEQ ${ }^{\circledR}$

Se nós tivéssemos um piso salarial e fossemos valorizados como profissionais não estaríamos com essa dupla jornada de trabalho (E10)

Eu já tentei sair de um emprego, mas você não sai com medo, porque você não sabe quando pode perder o outro, você fica à mercê, se você sai de um e perde o outro, como vai fazer se ficar sem nada? Você se sente ameaçado o tempo todo (T7)

Eu sempre penso em parar, mas não dá. Não dá para ficar só em um emprego, não dá para eu me manter e manter minha família (T11)

Eu quero estudar. Enfermagem não! Porque é pouco valorizada, paga pouco, então eu prefiro não ir para essa área porque eu sei que se eu for, para ter uma condição melhor, eu vou ter que acabar tendo dois empregos de novo. Então, eu ia continuar na mesma coisa (T2)

A segunda refere-se às repercussões da dupla jornada de trabalho apontando os aspectos negativos à saúde física, mental e social dos trabalhadores, que: "não dormem bem", "não se alimentam bem", "não fazem atividades físicas", "não tem tempo", "não tem vida social", "não vivem saudavelmente", "não querem demonstrar fraqueza".

Minha família diz que eu não vou aguentar, afinal tem vezes que a gente não dorme, come mal, não tem tempo pra fazer uma atividade física. Mas, eu sou forte! (T1)
Alguns colegas começaram com dupla jornada de trabalho e não deram conta por queixar de cansaço; outros não assumem porque já dizem que não dão conta (E1)

Eu não tenho tempo para fazer as coisas que eu gosto, para estar com quem eu gosto, eu acho que a gente deixa um pouco de viver (T20)

Você não tem tempo, você não tem vida social, você vive cansado e esse cansaço trás irritabilidade, problemas psicológicos, eu não vejo vantagem (T14)

Eu procuro não demonstrar, mas a dupla jornada de trabalho me afeta diretamente, me cansa, me desgasta (E2)

A maioria das pessoas que eu conheço que tem dois vínculos não vive saudável e as que vivem saudável se esforçam muito para ir no médico, por exemplo, só se for coisa de urgência porque na rotina não vai não (T4)

No tocante a palavra "gente" teve o sentido de coletividade (trabalhadores de enfermagem), que compartilham juntos as "dificuldades" que decorrem da dupla jornada de trabalho, a exemplo:

A gente começa ouvir dos colegas: está difícil, está puxado, não estou dando conta, aí chega uma hora que a gente pifa, aí vai ter que parar de qualquer jeito (E1)

$A$ renda da gente é muito baixa. Por isso, que a gente precisa ter dois empregos, não tem como ter só um. $A$ gente ganha pouco, a gente trabalha e se dedica muito 
e ganha pouco? Pra gente sobreviver só da enfermagem tem que ter dois empregos (T8)

A gente tem limites e principalmente quem faz plantões noturnos, porque a gente que faz plantões noturnos é muito diferente (E2)

\section{DISCUSSÃO}

A análise sobre o perfil dos trabalhadores participantes da pesquisa em relação ao sexo, gênero e autodeclaração de cor/raça, corroboram com o identificado em outras pesquisas e estabelecem relação com a trajetória histórica da profissão: predominantemente feminina e que se autodeclara em relação a cor/raça preta ou parda. ${ }^{11}$

Por se tratar de profissionais relativamente jovens, entende-se sua inserção em um contexto marcado pela desregulamentação das relações de trabalho e de precarização laboral, pois há, cada vez mais, um exército de reserva de mão de obra ávido para se inserir no mercado de trabalho devido à falta de postos de trabalho, mesmo que seja de forma indigna. Assim, apesar desse trabalho demandar uma alta carga horária semanal, com salários extremamente baixos, sob condições precarizadas, ainda é melhor que ficar desempregado. ${ }^{11-13}$

É possível considerar que os trabalhadores de enfermagem não escolheram por prazer vivenciar uma dupla jornada de trabalho, mas sentem-se forçados a adotá-la, diante dos baixos salários que recebem, da desvalorização da categoria e dos vínculos de trabalhos precários, temporários. Esse contexto de precarização do trabalho atende às necessidades do capitalismo e reflete a influência do modelo neoliberal sobre o processo de trabalho da enfermagem. 3,4,7-9

A precarização do trabalho é um processo decorrente da evolução do capitalismo moderno, que diante das novas exigências tecnológicas e econômicas, caracteriza-se pela desestabilização dos trabalhadores estáveis, a instalação da precariedade de diferentes trabalhadores e o aumento do desemprego, além da falta de proteção ao trabalhador e a baixa remuneração. ${ }^{13,14}$

Também é preciso considerar, que a incerteza da permanência no trabalho, o atual quadro de desemprego, especialmente entre enfermeiros, os vínculos laborais informais e instáveis, que levam os trabalhadores a permanecerem em mais de um emprego, a fim de garantir a subsistência material e um futuro mais estável economicamente, reforça o desgaste e o sofrimento psicofísico dos trabalhadores, tendo, assim, potencial ainda maior para o adoecimento do coletivo profissional. ${ }^{8}$

Infelizmente, esse contexto de desencantamento com as oportunidades do mercado, e com o mundo do trabalho, que se mostra perverso e excludente, pode culminar na evasão do profissional em relação a área de atuação, o que é bem negativo e afeta a subjetividade do trabalhador, pois ele mobilizou todo o seu esforço em prol da enfermagem, mas não vem obtendo a retribuição material e simbólica esperada. ${ }^{15,16}$
Sem a retribuição material, que se dá a partir do salário e das gratificações, e sem a retribuição simbólica; o reconhecimento e o prazer no trabalho pode ser transformado em sofrimento, na medida em que não se estabelece a dinâmica de realização do eu no contexto do trabalho. ${ }^{5}$

Todavia, é preciso ponderar que, nesse contexto, onde prevalece a desvalorização e a falta de reconhecimento ao trabalhador, o desempenho pode ser afetado negativamente. Pois, se os processos de reconhecimento e valorização são fontes mobilizadoras, capazes de aumentar o comprometimento e satisfação do trabalhador com o serviço, capazes inclusive, de repercutir em uma melhor qualidade do cuidado, o oposto pode gerar impactos negativos. ${ }^{17}$

As duplas jornadas de trabalho reduzem o tempo livre do trabalhador, o que pode afetar a sua vida pessoal, familiar e social. Essa situação tende a ser agravada entre as mulheres. Afinal, a concepção de que pessoas do sexo feminino se configuram como protagonistas do trabalho doméstico, do cuidado do lar/família e da criação dos filhos, ainda é muito presente na sociedade ocidental contemporânea, mesmo sabendo que alguns homens vêm mudando sua postura no sentido de posicionarem-se de maneira proativa e participativa na vida e na rotina familiar. ${ }^{18}$

A falta de tempo e a sobrecarga de funções domésticas e profissionais são os fatores mais comuns geradores de negligência com a saúde e impeditivos à adoção de comportamentos preventivos de agravos e de promoção da saúde, destacando-se o sedentarismo, a má alimentação, o padrão de sono/repouso insuficiente, a não realização de exames, com consequências negativas à saúde e bem-estar. ${ }^{4}$

A alteração no padrão de sono, pode produzir distração, comportamento automático, lapsos involuntários de sono e amnésia e, assim, os trabalhadores de enfermagem podem comprometer a assistência prestada aos pacientes sob sua responsabilidade. ${ }^{19}$ Tal situação demonstra que a carga de trabalho desses profissionais é um dos fatores que pode comprometer a segurança do paciente, pois mesmo diante da vigilância e preocupação dos trabalhadores em relação aos cuidados desenvolvidos, os riscos decorrentes da falha humana, podem se sobrepor.

Soma-se a essa conjuntura, o fato dos trabalhadores de enfermagem com dupla jornada de trabalho, apesar de sentirem fisicamente o cansaço e outras repercussões dessa prática sobre à saúde, negam o sofrimento, negligenciam os sinais emitidos pelo corpo cansado, e adotam uma postura de resiliência, e, até mesmo presenteísta.

O presenteísmo é caracterizado pela presença física do profissional no ambiente de trabalho em condições biológicas e psíquicas inoperantes para o exercício eficaz de sua atividade..$^{20} \mathrm{E}$ um problema emergente e preocupante, pois, além de diminuir a produtividade, é difícil de ser detectado. Assim, se, os problemas de saúde dos trabalhadores não são detectados precocemente, podem tornar-se crônicos e incapacitantes, o que interfere na qualidade de vida do trabalhador. ${ }^{21}$ 
O comportamento presenteísta entre os trabalhadores de enfermagem com dupla jornada de trabalho pode estar relacionada a empatia, cordialidade, solidariedade e identificação entre os profissionais, indicando que se reconhecem como pertencentes a um mesmo grupo, uma "gente" que sofre em decorrência do trabalho e da precarização do trabalho da enfermagem.

Todavia, há que se considerar, que o presenteísmo afeta negativamente $o$ ambiente de trabalho, e essa situação nem sempre é percebida pelo trabalhador, gestores e equipe de trabalho, mas, interfere no bem-estar e na qualidade de vida do trabalhador, bem como gera a desqualificação da assistência. ${ }^{20,21}$

Assim, há necessidade de mudança nos contextos de trabalho da enfermagem e no tocante a prática da dupla jornada de trabalho, devido ao seu potencial de causar adoecimento aos trabalhadores e, consequentemente, prejudicar a assistência prestada. É preciso, a essa categoria, libertar-se das amarras do passado, ${ }^{22}$ que a tornou subserviente e conformada e assumir o papel de proprietária do seu destino, sob pena de ver ser degradado um ofício tão nobre, que faz a diferença na vida de quem nasce, se desenvolve, se reproduz e morre.

\section{CONCLUSÃO E IMPLICAÇÕES PARA PRÁTICA}

O estudo permitiu conhecer por meio da lexicografia básica, os vocabulários mais frequentes no relato do cotidiano de trabalho dos trabalhadores de enfermagem com dupla jornada de trabalho. A palavra não foi a mais frequente, deixando em evidência a negatividade, restrições e sofrimento vivenciado pelos participantes em virtude de sua dinâmica de trabalho. Tal fato, coloca em risco a saúde e a subjetividade desse coletivo de trabalhadores bem como suas relações sociais e a qualidade da assistência.

$\mathrm{E}$, sendo o trabalho de enfermagem tão essencial aos serviços de saúde e à população, é fundamental, imprescindível e urgente repensar sobre a prática da dupla jornada de trabalho, para que esses trabalhadores possam reelaborar sua relação com o trabalho e, por meio de uma relação positiva, ampliar suas vivências de prazer no trabalho. Ademais, também é mister que essa gente (trabalhadores de enfermagem) possa se reconhecer e se fortalecer como classe trabalhadora e, assim, contribuir para a construção de políticas públicas voltadas à valorização e proteção dos trabalhadores de enfermagem.

Entende-se como limitação deste estudo o fato de discutir apenas os principais léxicos evocados pelos trabalhadores de enfermagem com dupla jornada de trabalho e que a nuvem de palavras permite uma análise lexical simples. Todavia, ressaltase que esse tipo de análise pode ser complementado por meio dos outros recursos disponíveis no IRAMUTEQ ${ }^{\circledR}$, ou seja, os dados podem ser analisados, por exemplo, por meio de análises multivariadas, sugerindo-se, portanto, o seu desenvolvimento, a fim de confirmar os resultados ora apresentados e ampliar as discussões sobre a temática. A replicação deste estudo em outros contextos também é importante no sentido de validar os achados.
Cabe destacar que o software IRAMUTEQ ${ }^{\circledR}$ se mostrou uma ferramenta importante para realização deste estudo. E, tendo em vista que ainda são poucos os artigos, no Brasil, que utilizam esse software e o método de análise produzido pela nuvem de palavras, este estudo contribui ao divulgar essa possiblidade de análise dos dados qualitativos.

\section{FINANCIAMENTO}

"O presente trabalho foi realizado com apoio da Coordenação de Aperfeiçoamento de Pessoal de Nível Superior - Brasil (CAPES) - Código de Financiamento 001, bolsa de mestrado, concedida a Samira Silva Santos Soares (processo oㅜ 88887.200750/2018-00).

\section{CONTRIBUIÇÕES DOS AUTORES}

Desenho do estudo. Samira Silva Santos Soares. Marcia Tereza Luz Lisboa

Coleta ou produção dos dados. Samira Silva Santos Soares Análise de dados. Samira Silva Santos Soares. Marcia Tereza Luz Lisboa. Ana Beatriz Azevedo Queiroz. Karla Gualberto Silva. Jandra Cibele Rodrigues de Abrantes Pereira Leite. Norma Valéria Dantas de Oliveira Souza

Interpretação dos resultados. Samira Silva Santos Soares. Marcia Tereza Luz Lisboa. Ana Beatriz Azevedo Queiroz. Karla Gualberto Silva. Jandra Cibele Rodrigues de Abrantes Pereira Leite. Norma Valéria Dantas de Oliveira Souza

Redação e revisão crítica do manuscrito. Samira Silva Santos Soares. Marcia Tereza Luz Lisboa. Ana Beatriz Azevedo Queiroz. Karla Gualberto Silva. Jandra Cibele Rodrigues de Abrantes Pereira Leite. Norma Valéria Dantas de Oliveira Souza

Aprovação da versão final do artigo. Samira Silva Santos Soares. Marcia Tereza Luz Lisboa. Ana Beatriz Azevedo Queiroz. Karla Gualberto Silva. Jandra Cibele Rodrigues de Abrantes Pereira Leite. Norma Valéria Dantas de Oliveira Souza

Responsabilidade por todos os aspectos do conteúdo e a integridade do artigo publicado. Samira Silva Santos Soares. Marcia Tereza Luz Lisboa. Ana Beatriz Azevedo Queiroz. Karla Gualberto Silva. Jandra Cibele Rodrigues de Abrantes Pereira Leite. Norma Valéria Dantas de Oliveira Souza

\section{EDITOR ASSOCIADO}

Aline Aparecida Monroe

\section{REFERÊNCIAS}

1. Marx K. O Capital. São Paulo: Nova Cultural; 1996. Os Economistas, livro I, tomo 2.

2. Delgado MG. Curso de Direito do Trabalho. 9ª ed. São Paulo: LTr; 2010.

3. Gomes HF, Gonçalves FGA, Pires AS, Jomar RT, Peres EM, Gallasch CH. Reflection of nursing labor precarization in brazil for nursing workforce health status: an integrative review. Rev Enferm Atual Derme. 2016;16(77):6774. http://dx.doi.org/10.31011/reaid-2016-v.77-n.15-art.376.

4. Albuquerque GA, Nunes JFC, Belem JM, Leite MF, Quirino GDS Double work shift: implications on nurses' health. Rev. Enfermagem UFPE online. 2016;10(9):3401-10. http://dx.doi.org/10.5205/reuol.957183638-1-SM1009201628. 
5. Dejours C, Abdoucheli E, Jayet C, Betiol MIS. Psicodinâmica do trabalho: contribuições da Escola Dejouriana à Análise da Relação prazer, sofrimento e trabalho. São Paulo: Atlas; 2007.

6. Pimenta GF, Perez Jr EF, Pires ADS, Gomes HF, Thiengo PCS, Paula VG. Influência da precarização no processo de trabalho e na saúde do trabalhador de enfermagem. Rev. Enferm. UERJ [Internet]. 2013; [citado 2018 maio 28];21(2):265-8. Disponível em: http://www.e-publicacoes. uerj.br/index.php/enfermagemuerj/article/view/7224

7. Pimenta GF, Pérez Jr EF, Pires AS, Gomes HF, Thiengo PCS, Paula VG. Influence of precariousness in the nursing work process and in the worker's health. Rev Enferm UFSM. 2018;8(4):758-68. http://dx.doi. org/10.5902/2179769230180.

8. Dias MO, Souza NVDO, Penna LHG, Gallasch CH. Perception of nursing leadership on the fight against the precariousness of working conditions. Rev Esc Enferm USP. 2019;53:e03492. http://dx.doi.org/10.1590/ s1980-220x2018025503492. PMid:31433021.

9. Shoji S, Souza NVDO, Farias SNP. Impact of workplace on the health of nursing professionals at a specialized outpatient clinic. Reme: Rev Min Enferm. 2015;19(1):43-8. http://dx.doi.org/10.5935/1415-2762.20150004.

10. Camargo BV, Justo AM. Tutorial para uso do software de análise textual IRAMUTEQ [Internet]. Florianópolis: Universidade Federal de Santa Catarina; 2018 [citado 2019 mar 20]. Disponível em: http://www. iramuteq.org/documentation/fichiers/tutoriel-portugais-22-11-2018

11. Machado MH, Oliveira E, Lemos W, Lacerda WS, Aguiar WF, Wermelinger MV et al. Mercado de trabalho da enfermagem: aspectos gerais. Enferm. Foco. [Internet]. 2016; [citado 2019 ago 25];7(N. esp.):35-62. Disponível em: http://biblioteca.cofen.gov.br/mercado-de-trabalho-da-enfermagemaspectos-gerais

12. Gonçalves FGA, Souza NVDO, Zeitoune RC, Adame GF, Nascimento SM. Impacts of neoliberalism on hospital nursing work. Texto Contexto Enferm. 2015;24(3):646-53. http://dx.doi.org/10.1590/0104-07072015000420014.

13. Oliveira BLCA, Silva AM, Lima SF. Carga semanal de trabalho para enfermeiros no Brasil: desafios ao exercício da profissão. Trab. Educ.
Saúde. 2018;16(3):1221-36. http://dx.doi.org/10.1590/1981-7746sol00159.

14. Oliveira EA. A funcionalidade da política social e os limites da emancipação humana na ordem do capital. SER Social. 2015;17(37):348-67. http:// dx.doi.org/10.26512/ser_social.v17i37.13436.

15. Castel R. Precarização do trabalho. In: Mendes R, organizador. Dicionário de saúde e segurança do trabalhador: conceitos, definições, história, cultura. Novo Hamburgo: Proteção Publicações; 2018. p. 1030-3.

16. Vieira ML, Oliveira EB, Souza NVDO, Lisboa MT, Xavier T, Rossone FO. Job insecurity at a teaching hospital and presenteeism among nurses. Rev enferm UERJ. 2016;24(4):e23580. http://dx.doi.org/10.12957/ reuerj.2016.23580.

17. Lages CEB, Alves MS. (Des)valorização da enfermagem: implicações no cotidiano do enfermeiro. Enfermagem em foco 2016;7(3/4):12-6. https://doi.org/10.21675/2357-707X.2016.v7.n3/4.908.

18. Rodrigues BC, Lima MF, Maschio B No, Oliveira GL, Corrêa ACP, Higarashi IH. Being a mother and a nurse: issues about gender and overlapping social roles. Rev. Rene. 2017;18(1):91-8. http://dx.doi. org/10.15253/2175-6783.2017000100013.

19. Soares CG, Mello MCVA, Santos KNSC, Modernel DX, Cezar-Vaz MR Excessive daytime sleepiness among nursing professionals. Rev enferm UFPE on line. 2018;12(6):1603-9. https://doi.org/10.5205/1981-8963v12i6a231336p1603-1609-2018.

20. Umann J, Guido LA, Grazziano ES. Presenteeism in hospital nurses. Rev Lat Am Enfermagem. 2012;20(1):159-66. http://dx.doi.org/10.1590/ S0104-11692012000100021. PMid:22481734.

21. Silva BMCCS, Lucca SR. Presenteísmo no Trabalho. In: Mendes R, organizador. Dicionário de saúde e segurança do trabalhador: conceitos, definições, história, cultura. Novo Hamburgo: Proteção Publicações; 2018.

22. Ferreira ICA. Condições de trabalho da enfermagem. Enferm. Foco. 2016;7(N. esp.):63-76. https://doi.org/10.21675/2357-707X.2016. v7.nESP.697.

\footnotetext{
a Extraído da dissertação de mestrado "Dupla Jornada de Trabalho: Repercussões para a saúde doTrabalhador de Enfermagem", de autoria de Samira Silva Santos Soares, sob a orientação de Marcia Tereza Luz Lisboa, apresentada ao Programa de Pós-Graduação em Enfermagem, da Escola de Enfermagem Anna Nery, em 2020.
} 